\title{
Avaliação de Formulações de Glyphosate sobre Soja RounduP READY $^{1}$
}

\author{
Effects of Glyphosate Formulations on Transgenic Soybean
}

\author{
SANTOS, J.B. ${ }^{2}$, FERREIRA, E.A. ${ }^{3}$, REIS, M.R. ${ }^{4}$, SILVA, A.A. ${ }^{5}$, FIALHO, C.M.T. ${ }^{6}$ e \\ FREITAS, M.A.M. ${ }^{6}$
}

\begin{abstract}
RESUMO - Avaliou-se neste trabalho o efeito da aplicação de três marcas comerciais de glyphosate - Roundup Ready ${ }^{\circledR}$ e R. Transorb ${ }^{\circledR}$, formuladas à base do sal de isopropilamina, e Zapp Qi ${ }^{\circledR}$, à base do sal potássico - sobre a soja transgênica (variedade CD 219RR), tolerante a esse herbicida. Aos 25 dias após a emergência, quando as plantas apresentavam o segundo trifólio completamente expandido (estádios $\mathrm{V}_{2}-\mathrm{V}_{3}$ ), foram aplicadas formulações do glyphosate na dose de $2.000 \mathrm{~g} \mathrm{ha}^{-1}$. Avaliou-se a intoxicação das plantas aos 15 dias após a aplicação do herbicida, o número e massa seca de folíolos, o número de nódulos radiculares e o teor foliar de N, P, K, S, Ca, Mg, Fe, Cu, Zn e Mn, por ocasião do florescimento (59 dias após a emergência - DAE), e o rendimento de grãos ao final do ciclo (127 DAE). A partir de amostras de solo, também coletadas na etapa de florescimento da soja, avaliou-se a taxa de respiração basal do solo, o carbono da biomassa microbiana e quociente metabólico. O Roundup Transorb, formulado à base do sal de isopropilamina, foi mais prejudicial às plantas de soja RG, reduzindo o número de nódulos radiculares e o rendimento de grãos, além de promover maior efeito negativo sobre a microbiota do solo. Roundup Ready, à base do mesmo sal isopropilamina, não deve ser aplicado na soja RG em doses elevadas, haja visto ser capaz de alterar o teor de alguns nutrientes nas folhas da cultura, entre eles $\mathrm{N}, \mathrm{Ca}, \mathrm{Mg}, \mathrm{Fe}$ e $\mathrm{Cu}$, além de causar intoxicação nas plantas.
\end{abstract}

Palavras-chave: herbicidas, seletividade, soja transgênica, inibidores da EPSPs.

\begin{abstract}
This study aimed to evaluate the effects of three glyphosate formulations (Roundup Ready ${ }^{\circledR}$ and $R$. Transorb ${ }^{\circledR}$ - both with isopropylamine salt and Zapp Q ${ }^{\circledR}$, formulated as potassium salt), on transgenic soybean. CD 219RR variety soybean plants displaying the CP4Epsps gene, tolerant to glyphosate, were cultivated. At 25 days after emergence (DAE), when plants showed the second trifolium completely expanded (stadiums $V_{2}-V_{3}$ ), formulations were applied at 2,000 $\mathrm{g} \mathrm{ha}^{-1}$. Plants intoxication was evaluated 15 days after application as well as the number and dry matter of leaflets, number of radicular nodules and foliar content of $N, P, K, S, C a, M g$, $\mathrm{Fe}, \mathrm{Cu}, \mathrm{Zn}$, and $\mathrm{Mn}$ at flowering and grain yield at the end of the cycle. Soil basal respiration rate, microbial biomass carbon and metabolic quotient were evaluated through soil samples collected during soybean flowering. Isopropylamine salt, present in the Roundup Transorb formulation, was more harmful to the soybean plants, also providing a negative effect on the soil microbiota. Roundup Ready formulation, registered as transgenic soybean, should not be applied on this crop at a higher rate, since it could alter the content of some nutrients, such as $N$, $\mathrm{Ca}, \mathrm{Mg}, \mathrm{Fe}$ and $\mathrm{Cu}$, besides causing intoxication in the plants.
\end{abstract}

Keywords: herbicides, selectivity, transgenic soybean, EPSPs inhibitors.

Recebido para publicação em 9.9.2006 e na forma revisada em 27.2.2007.

Doutor em Produção Vegetal, Professor de Agronomia da Faculdade de Ciências Agrárias, Universidade Vale do Rio Doce FAAG/UNIVALE, Rua Israel Pinheiro, 2000, Bairro Universitário, 35020-220 Governador Valadares-MG, <jbarbosa@univale.br>. ${ }^{3}$ Bolsista de Doutorado, CNPq, Dep. de Fitotecnia da Universidade Federal de Viçosa - DFT/UFV, 36570-000 Viçosa-MG; ${ }^{4}$ Bolsista de Mestrado, CNPq, Dep. de Fitotecnia - DFT/UFV; ${ }^{5}$ Prof. Associado - DFT/UFV; ${ }^{6}$ Bolsistas de Iniciação Científica - FAPEMIG e CNPq, respectivamente. 


\section{INTRODUÇÃO}

Com o desenvolvimento da soja geneticamente modificada, resistente ao glyphosate, tem-se observado aumento considerável na utilização desse herbicida aplicado, também, em pós-emergência dessa cultura.

O herbicida glyphosate inibe a sintese dos aminoácidos aromáticos por atuar na enzima precursora EPSPs (5 enolpiruvilchiquimato-3fosfato sintase), evitando a transformação do chikimato em corismato (Shaner \& Bridges, 2003). No caso da soja resistente ao glyphosate, a tolerância ao herbicida foi obtida pela inserção de um gene (AroA) oriundo do genoma da Agrobacterium sp., estirpe CP4, a qual codifica uma variante da EPSPs (CP4 EPSPS), especialmente tolerante à inibição pelo glyphosate (Padgette et al., 1995). Sob tratamento com esse herbicida, as plantas de soja não são afetadas, em virtude da ação continuada e sistemática dessa enzima alternativa, insensivel ao produto.

No entanto, há relatos de agricultores sobre o possivel efeito do glyphosate afetando negativamente o desenvolvimento inicial de plantas de soja, para a qual esse produto é recomendado. Esse efeito pode estar relacionado ao aumento demasiado da dose aplicada, à aplicação de outras formulações de glyphosate não recomendadas para a cultura, ou ao efeito de outras substâncias químicas naturais ou sintéticas, como aleloquímicos ou surfatantes, respectivamente. Atualmente são disponibilizadas no mercado diversas formulações de glyphosate, que, apesar de apresentarem o mesmo mecanismo de ação, possuem, na composição, diferentes sais, sendo os principais: sal potássico, de isopropilamina e de amônio (Rodrigues \& Almeida, 2005).

As particularidades de cada formulação incluem maior intoxicação a organismos não-alvo, principalmente para a microbiota do solo (Santos et al., 2005, 2006), maior velocidade de translocação e de ação (Molin \& Hirase, 2005), melhor controle de algumas espécies de plantas daninhas (Jakelaits et al., 2001; Molin \& Hirase, 2004; Li et al., 2005) e desbalanço no estado nutricional das plantas (Duke et al., 1983).

A avaliação do estado nutricional das plantas geralmente é realizada pela diagnose foliar, considerando-se que a folha recém-madura é o órgão que geralmente mais responde às variações no suprimento do nutriente, pela adição ou já presente no solo (Malavolta et al., 1997). É também nas folhas que ocorrem as principais reações metabólicas, onde as alterações fisiológicas decorrentes de distúrbios nutricionais geralmente se tornam mais evidentes (Martin-Prevel et al., 1984). A determinação dos teores de nutrientes como critério de diagnose baseia-se na premissa de existir relação significativa entre o suprimento destes e os seus teores no tecido amostrado, bem como entre os teores e as produções das culturas (Evenhuis \& Waard, 1980).

De maneira geral, há certa concordância para os níveis de suficiência de nutrientes na cultura da soja (Sfredo et al., 1986; Malavolta et al., 1997; Embrapa, 2002).

Com relação aos efeitos negativos do glyphosate sobre a soja transgênica, resistente a esse herbicida, é possivel atribuir a causa a adjuvantes presentes na formulação ou ao tipo de sal presente (Pline et al., 1999; Reddy \& Zablotowicz, 2003). Os surfatantes, para promoverem maior intoxicação visual do glyphosate sobre as plantas, são requeridos em concentrações maiores que as necessárias para reduzirem a tensão superficial, indicando que o modo de ação não está só limitado à sua ação como espalhante das gotas, mas poderiam atuar no aumento da permeabilidade da cutícula ou da membrana celular, ou de ambas (Sherrick et al., 1986).

Stock \& Holloway (1993) afirmaram que os surfatantes, além de melhorarem a deposição e retenção do herbicida, podem penetrar na cutícula, às vezes rapidamente, para atingir outros lugares de ação, como a membrana celular, aumentando sua permeabilidade em baixas concentrações ou danificando-a em maiores concentrações. Riechers et al. (1994) observaram que a eficácia dos surfatantes para incrementar a absorção do glyphosate depende da habilidade de se difundir através da cutícula para chegar ao apoplasto e atuar diretamente na membrana celular com a proteína ou com os lipídeos, aumentando sua permeabilidade.

Com o presente trabalho, objetivou-se avaliar o efeito de três formulações de glyphosate sobre a soja transgênica, assim como o impacto desse herbicida na microbiota do solo. 


\section{MATERIAL E MÉTODOS}

Os experimentos foram realizados em casa de vegetação e laboratórios pertencentes ao Departamento de Fitotecnia da UFV.

Para realização deste trabalho cultivaramse plantas de soja da variedade CD 219RR com o gene CP4EPSPS, tolerante ao glyphosate (RG). As plantas foram cultivadas em vasos com capacidade para seis litros, com substrato previamente preparado para seu adequado des envolvimento (Tabela 1). Semearam-se quatro sementes por vaso, sendo posteriormente realizado o desbaste das plântulas, deixando-se duas plantas por vaso.

Aos 25 dias após a emergência (DAE), quando as plantas de soja apresentavam o segundo trifólio completamente expandido (estádios $\mathrm{V}_{2}-\mathrm{V}_{3}$ ), foram dispostos os tratamentos compostos pela aplicação de três formulações

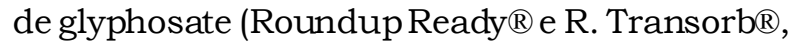
ambas contendo o sal de isopropilamina, e Zapp Qi®, formulado à base do sal potássico), aplicadas na dose de $2.000 \mathrm{~g} \mathrm{ha}^{-1}$ do equivalente ácido, mais uma testemunha sem aplicação de herbicida, cada um com oito repetições. $\mathrm{Na}$ aplicação dos herbicidas utilizou-se um pulverizador costal pressurizado com gás carbônico $\left(\mathrm{CO}_{2}\right)$, aplicando-se o equivalente a $200 \mathrm{~L} \mathrm{ha}^{-1}$ de calda.

Aos 15 dias após a aplicação dos herbicidas, realizou-se a avaliação de intoxicação das plantas, atribuindo-se notas variando de zero (sem sintomas) a cem (morte total da planta). No estádio do florescimento $\left(R_{1}-\right.$ terceiro trifólio a partir do ápice), quatro repetições de cada tratamento foram colhidas para as avaliações de teor de nutrientes (Embrapa, 2002), peso e número de folíolos e número de nódulos radiculares. Também nessa etapa foram coletadas amostras do solo para as avaliações da atividade microbiológica.

As folhas, depois de coletadas, foram secas em estufa com circulação forçada de ar à temperatura de $65^{\circ} \mathrm{C}$, sendo após transferidas para o Laboratório de Nutrição Mineral de Plantas (DFT/UFV), onde foram moídas e submetidas à digestão nítrico-perclórica. Utilizaram-se amostras de $0,5 \mathrm{~g}$ em $10 \mathrm{~mL}$ de $\mathrm{HNO}_{3}$ concentrado, submetidas à temperatura de, no máximo, $200^{\circ} \mathrm{C}$ para a digestão. Após obtenção do extrato, determinou-se a concentração de fósforo $(\mathrm{P})$, de enxofre $(\mathrm{S})$, de potássio $(\mathrm{K})$, de cálcio $(\mathrm{Ca})$, de magnésio $(\mathrm{Mg})$, de zinco $(\mathrm{Zn})$, de ferro $(\mathrm{Fe})$, de manganês $(\mathrm{Mn})$ e de cobre $(\mathrm{Cu})$. Para determinação do nitrogênio orgânico ( $\mathrm{N}$ orgânico), as amostras foram submetidas à digestão sulfúrica.

A respiração microbiana (taxa de respiração basal do solo) foi estimada a partir da quantidade de $\mathrm{CO}_{2}$ evoluído de amostras de $100 \mathrm{~g}$ do solo coletadas em cada vaso, o qual foi capturado em frascos com $100 \mathrm{ml}$ de $\mathrm{NaOH}$ $\left(0,25 \mathrm{~mol} \mathrm{~L}^{-1}\right)$, em sistema contínuo de fluxo de ar (isento de $\mathrm{CO}_{2}$ e umidade). As amostras de solo, após serem passadas por peneira com malha de 2 mesh, secas ao ar e determinado o teor de água, foram pesadas e incubadas por sete dias em erlenmeyer com teor de água a $70 \%$ da capacidade de campo. Após incubação por 10 dias, procedeu-se à titulação indireta do hidróxido de sódio com $\mathrm{HCl}\left(0,25 \mathrm{~mol} \mathrm{~L}^{-1}\right)$, e o excesso de $\mathrm{NaOH}$ que não reagiu com o $\mathrm{CO}_{2}$ evoluído foi quantificado (Anderson, 1982).

Tabela 1 - Composição química e textural das amostras de solo utilizadas no cultivo de plantas de soja transgênica. Viçosa-MG, 2005

\begin{tabular}{|c|c|c|c|c|c|c|c|c|c|c|}
\hline \multicolumn{11}{|c|}{ Fração mineral (\%) } \\
\hline \multicolumn{2}{|c|}{ Areia fina } & \multicolumn{2}{|r|}{ Areia grossa } & \multicolumn{3}{|c|}{ Silte } & \multicolumn{2}{|l|}{ Argila } & \multicolumn{2}{|c|}{ Textura } \\
\hline \multicolumn{2}{|c|}{9} & \multicolumn{2}{|r|}{14} & \multicolumn{3}{|c|}{2} & \multicolumn{2}{|l|}{75} & \multicolumn{2}{|c|}{ Argilosa } \\
\hline \multicolumn{11}{|c|}{ Análise Química } \\
\hline $\mathrm{pH}$ & $\mathrm{P}$ & $\mathrm{K}+$ & $\mathrm{H}+\mathrm{Al}$ & $\mathrm{Al}^{3+}$ & $\mathrm{Ca}^{2+}$ & $\mathrm{Mg}^{2+}$ & $\mathrm{CTC}_{\text {total }}$ & $\mathrm{V}$ & $\mathrm{M}$ & $\mathrm{MO}$ \\
\hline$\left(\mathrm{H}_{2} \mathrm{O}\right)$ & \multicolumn{2}{|c|}{$\left(\mathrm{mg} \mathrm{dm}^{-3}\right)$} & \multicolumn{5}{|c|}{$\left(\mathrm{cmol}_{\mathrm{c}} \mathrm{dm}^{-3}\right)$} & \multicolumn{2}{|c|}{$(\%)$} & $\left(\operatorname{dag~kg}{ }^{-1}\right)$ \\
\hline 4,6 & 2,8 & 7,0 & 3,10 & 0,0 & 0,4 & 0,1 & 3,2 & 0,60 & 0,0 & 1,90 \\
\hline
\end{tabular}


Ao final do período de incubação das amostras de solos, determinaram-se o carbono da biomassa microbiana ( $\mathrm{CBM})$, pelo método descrito por Vance et al. (1987), utilizando-se, em lugar do clorofórmio (fumigação), forno de microondas (irradiação) (Islam \& Weil, 1998), e o quociente metabólico $\left(\mathrm{qCO}_{2}\right)$, por meio da relação entre o $\mathrm{CO}_{2}$ acumulado $\left(\mu \mathrm{g} \mathrm{g}^{-1}\right)$ e o C total da biomassa microbiana $\left(\mu \mathrm{g} \mathrm{g}^{-1}\right)$.

Ao final do ciclo, coletaram-se as demais repetições para determinação do rendimento de grãos da soja.

Os dados foram submetidos à análise de variância pelo teste $\mathrm{F}$, e as médias dos tratamentos quando significativas, para as características avaliadas, foram comparadas pelo teste de Tukey a $5 \%$ de probabilidade.

\section{RESULTADOS E DISCUSSÃO}

Comprovou-se o efeito variável do glyphosate sobre o desenvolvimento da soja, tolerante a esse produto, em função da formulação com que o produto comercial é produzido. Aos 15 dias após a aplicação das formulações, por meio da avaliação visual de sintomas de intoxicação, constatou-se leve efeito tóxico de Roundup Ready (25\%), seguido por Zapp Qi $(28 \%)$ e maior dano causado por Roundup Transorb (40\%) (Tabela 2).

Atingindo o florescimento, observou-se recuperação das plantas, porém, mesmo sem interferir no número e na massa seca de folíolos, Roundup Transorb diminuiu consideravelmente o número de nódulos radiculares produzidos na simbiose entre soja e rizóbio. Considerando a maior velocidade de absorção e translocação de Roundup Transorb, em relação às demais formulações encontradas no mercado à base de glyphosate, e levando-se em conta a sensibilidade do rizóbio da soja a esse herbicida, principalmente dessa formulação (Santos et al., 2005), é possivel que compostos presentes no produto comercial tenham promovido impacto negativo sobre o total de nódulos formados. Reddy \& Zablotowicz (2003) observaram que o sal de isopropilamina, presente também nas formulações $\mathrm{R}$. Transorb e $\mathrm{R}$. Ready, se acumulou nos nódulos radiculares da soja transgênica em concentrações bem acima das observadas para o sal trimetilsulfônico e diamônio.
O efeito de intoxicação, somado ao menor equilíbrio entre rizóbio e planta de soja, resultou em menor rendimento de grãos quando as plantas de soja foram tratadas com o Roundup Transorb. A formulação recomendada para aplicação nos cultivos de soja transgênica RG (Roundup Ready), apesar da tendência para a redução da produtividade, apresentou rendimento de grãos semelhante ao da testemunha (Tabela 2). É possivel que a maior contribuição para a redução na produtividade da soja, ocasionada pelas formulações de glyphosate, seja promovida pela intoxicação visual, a qual, entre outros prejuízos, afeta a capacidade da planta em produzir fotoassimilados.

Por meio da análise foliar na fase de florescimento da cultura, constatou-se diferença para alguns nutrientes (Tabelas 3 e 4). Para $\mathrm{N}, \mathrm{Ca}, \mathrm{Fe}$ e $\mathrm{Cu}$, observou-se diminuição quando as plantas foram tratadas com o glyphosate. Para o Mg, o efeito foi contrário, com aumento de seu teor nas folhas das plantas tratadas com o herbicida (Tabela 3). Especificamente para o nitrogênio, nas plantas de soja tratadas com R. Transorb, acredita-se que a diminuição da capacidade da cultura em realizar a simbiose com o rizóbio para formação dos nódulos tenha contribuído para o menor teor desse elemento nas folhas, uma vez que, para o plantio comercial dessa cultura, praticamente não se realiza a adubação nitrogenada, ou, quando se faz, é feita em baixíssimas doses. No entanto, diferentemente do observado para a capacidade de nodulação, a aplicação de qualquer uma das formulações do glyphosate promoveu a diminuição do teor de $\mathrm{N}$ nas folhas, levando-se a considerar que a aplicação desse herbicida, mesmo a recomendada para a cultura da soja, poderia esclarecer relatos de alguns agricultores sobre o insatisfatório desenvolvimento inicial de plantas de soja em cultivos sob sistema de plantio direto.

Semelhante ao observado para o N, o cálcio foi diminuído nas folhas das plantas tratadas com glyphosate. No entanto, para esse macronutriente, a aplicação de R. Transorb promoveu menor redução, comparado às demais formulações (Tabela 3). A maior redução do cálcio promovida pelas formulações de glyphosate pode ser atribuída à maior velocidade de penetração desses produtos nas plantas de soja. Duke et al. (1983) observaram 
Tabela 2 - Valores observados para número e massa seca de folíolos de plantas de soja resistentes ao glyphosate por ocasião do florescimento, intoxicação das plantas (15 dias após a aplicação dos herbicidas) e produtividade final de grãos após aplicação do herbicida em diferentes formulações, 25 dias após o plantio

\begin{tabular}{|c|c|c|c|c|c|}
\hline \multirow{2}{*}{ Tratamento } & $\begin{array}{c}\text { Massa seca de } \\
\text { folíolos }\end{array}$ & Folíolos & $\begin{array}{c}\text { Nódulos } \\
\text { radiculares }\end{array}$ & Intoxicação & Rendimento \\
\cline { 2 - 6 } & $(\mathrm{g})$ & \multicolumn{2}{|c|}{ (Número por planta) } & \multicolumn{2}{c|}{$(\%)$} \\
\hline Testemunha & 2,040 & $36,75 \mathrm{a}$ & $33,0 \mathrm{a}$ & $0 \mathrm{a}$ & $100,0 \mathrm{a}$ \\
\hline Roundup Ready & 1,794 & $34,50 \mathrm{ab}$ & $29,5 \mathrm{a}$ & $25 \mathrm{~b}$ & $93,45 \mathrm{ab}$ \\
\hline Roundup Transorb & 1,911 & $32,25 \mathrm{ab}$ & $18,3 \mathrm{~b}$ & $40 \mathrm{c}$ & $70,02 \mathrm{c}$ \\
\hline Zapp Qi & 1,943 & $30,75 \mathrm{~b}$ & $30,0 \mathrm{a}$ & $28 \mathrm{~b}$ & $81,60 \mathrm{bc}$ \\
\hline Média & $1,922^{\text {ns }}$ & - & - & - & - \\
\hline
\end{tabular}

Tabela 3 - Valores observados para teor de macronutrientes presentes nas folhas de soja resistente ao glyphosate, coletadas por ocasião do florescimento após aplicação desse herbicida em diferentes formulações, 25 dias após o plantio

\begin{tabular}{|c|c|c|c|c|c|c|c|}
\hline \multirow{2}{*}{ Tratamento } & $\mathrm{N}$ & $\mathrm{P}$ & $\mathrm{K}$ & $\mathrm{S}$ & \multicolumn{2}{c|}{$\mathrm{Ca}$} & $\mathrm{Mg}$ \\
\cline { 2 - 8 } & \multicolumn{7}{|c|}{$\left(\mathrm{g} \mathrm{kg}^{-1}\right)$} \\
\hline Testemunha & $36,41 \mathrm{a}$ & 1,20 & 16,32 & 10,90 & $11,44 \mathrm{a}$ & $2,81 \mathrm{~b}$ \\
\hline Roundup Ready & $27,95 \mathrm{~b}$ & 0,93 & 16,16 & 12,84 & $8,81 \mathrm{c}$ & $4,16 \mathrm{a}$ \\
\hline Roundup Transorb & $30,89 \mathrm{ab}$ & 1,01 & 18,52 & 11,97 & $10,12 \mathrm{~b}$ & $4,43 \mathrm{a}$ \\
\hline Zapp Qi & $29,16 \mathrm{~b}$ & 1,02 & 18,84 & 12,70 & $7,94 \mathrm{c}$ & $3,84 \mathrm{a}$ \\
\hline Média & - & $1,04^{\text {ns }}$ & $17,46^{\text {ns }}$ & $12,10^{\text {ns }}$ & - & - \\
\hline
\end{tabular}

diminuição no conteúdo de cálcio em plantas de soja tratadas com glyphosate. Esses autores verificaram que, na presença do glyphosate, o cálcio era transportado para raízes e hipocótilo; contudo, a partir de quatro dias da aplicação, a translocação desse nutriente para as folhas de soja era fortemente inibida na presença do herbicida.

Os teores de P, K, S, Zn e Mn não foram alterados por ocasião da aplicação dos herbicidas (Tabelas 3 e 4). Para o Mg, foi observado aumento no teor nas folhas amostradas (Tabela 3). Esse nutriente é requerido para formação da clorofila "a" unido por ligações coordenadas com os átomos de nitrogênio no anel de porfirina, além de ser necessário para várias enzimas na transferência de fosfato (Rao et al., 1987). É provável que a diminuição dos teores de nitrogênio, somada aos efeitos de intoxicação visual, tenha contribuído para o aumento da concentração desse nutriente nas folhas tratadas com o glyphosate.

Avaliando os bioindicadores da qualidade do solo, verificou-se efeito negativo da aplicação
Tabela 4 - Valores observados para teor de micronutrientes presentes nas folhas de soja resistente ao glyphosate, coletadas por ocasião do florescimento, após aplicação desse herbicida em diferentes formulações, 25 dias após o plantio

\begin{tabular}{|l|c|c|c|c|}
\hline \multirow{2}{*}{ Tratamento } & $\mathrm{Fe}$ & $\mathrm{Cu}$ & $\mathrm{Zn}$ & $\mathrm{Mn}$ \\
\cline { 2 - 6 } & \multicolumn{4}{|c|}{$\left(\mathrm{mg} \mathrm{kg}^{-1}\right)$} \\
\hline Testemunha & $643,23 \mathrm{a}$ & $7,98 \mathrm{a}$ & 58,08 & 142,94 \\
\hline Roundup Ready & $208,47 \mathrm{~b}$ & $4,45 \mathrm{~b}$ & 56,00 & 176,50 \\
\hline Roundup Transorb & $129,32 \mathrm{~b}$ & $4,05 \mathrm{~b}$ & 58,58 & 176,81 \\
\hline Zapp Qi & $96,08 \mathrm{~b}$ & $3,90 \mathrm{~b}$ & 49,55 & 138,00 \\
\hline \multicolumn{1}{|c|}{ Média } & - & - & $55,55^{\text {ns }}$ & $158,56^{\text {ns }}$ \\
\hline
\end{tabular}

do glyphosate, na formulação R. Transorb à base do sal de isopropilamina. Essa formulação foi a única, entre as testadas, a diminuir a atividade microbiana do solo (Tabela 5). Apesar de o herbicida, na formulação mencionada, não ter promovido mudanças na taxa de respiração basal do solo - $\mathrm{CO}_{2}$ desprendido das amostras de solo -, o total de carbono da biomassa microbiana $(\mathrm{CBM})$ foi diminuído para valor abaixo de $87 \mu \mathrm{g} \mathrm{g}^{-1}$ de solo quando, no solo da testemunha sem aplicação do herbicida, 
esse valor foi superior a $130 \mu \mathrm{g} \mathrm{g}^{-1}$ de solo (Tabela 5). A diminuição no total de microrganismos, representado pelo menor valor de CBM, sem alteração no desprendimento de $\mathrm{CO}_{2}$ pode representar menor eficiência de utilização do carbono nas reações metabólicas da microbiota do solo de maneira geral (Anderson \& Domsch, 1985). O quociente metabólico $\left(\mathrm{qCO}_{2}\right)$, estabelecido pela relação entre o $\mathrm{CO}_{2}$ acumulado e o total do CBM, indica que, com o aumento da eficiência dos microrganismos em utilizar os recursos em seu meio, menos $\mathrm{C}$ é perdido como $\mathrm{CO}_{2}$ pela respiração, podendo este ser incorporado aos tecidos microbianos (Anderson \& Domsch, 1985). Dessa forma, menor $\mathrm{qCO}_{2}$ significa maior estabilidade da biomassa microbiana, ou seja, maior estabilidade no sistema. Nesta pesquisa, o maior valor observado para $\mathrm{qCO}_{2}$ foi de 0,171 nas amostras de solo que receberam a aplicação $\mathrm{R}$ Transorb com o sal de isopropilamina, representando, em média, $72 \%$ acima dos valores observados nos demais tratamentos (Tabela 5). É provável que o efeito negativo dessa formulação aos microrganismos do solo seja o resultado dos efeitos negativos ocasionados pelos surfatantes presentes na composição do produto comercial, que podem agir por intoxicação direta aos microrganis mos; do efeito indireto, por facilitar maior absorção de glyphosate por uma grande parte de microrganismos que possuem em seu metabolismo a enzima EPSPsintase; ou por ambos os efeitos.

De maneira geral, pode-se afirmar que o Roundup Transorb formulado à base do sal de isopropilamina possui maior efeito negativo sobre plantas de soja tolerantes ao glyphosate,

Tabela 5 - Taxa diária de respiração basal do solo $\left(\mathrm{CO}_{2}\right)$, carbono da biomassa microbiana (CBM) e quociente metabólico $\left(\mathrm{qCO}_{2}\right)$ em amostras de solo cultivado com soja resistente ao glyphosate, coletadas por ocasião do florescimento, após aplicação desse herbicida em diferentes formulações, 25 dias após o plantio

\begin{tabular}{|l|c|c|c|}
\hline \multirow{2}{*}{ Tratamento } & $\mathrm{CO}_{2}$ & $\mathrm{CBM}$ & $\mathrm{qCO}_{2}$ \\
\cline { 2 - 4 } & \multicolumn{2}{|c|}{$\left(\mu \mathrm{g} \mathrm{g}^{-1}\right)$} & $\left(\mathrm{CO}_{2} \mathrm{CBM}^{-1}\right)$ \\
\hline Testemunha & 129,2 & $130,41 \mathrm{a}$ & $0,99 \mathrm{a}$ \\
\hline Roundup Ready & 112,6 & $116,21 \mathrm{a}$ & $0,97 \mathrm{a}$ \\
\hline Roundup Transorb & 147,8 & $86,80 \mathrm{~b}$ & $1,71 \mathrm{~b}$ \\
\hline Zapp Qi & 120,5 & $120,48 \mathrm{a}$ & $1,00 \mathrm{a}$ \\
\hline \multicolumn{1}{|c|}{ Media } & $12,75^{\text {ns }}$ & - & - \\
\hline
\end{tabular}

diminuindo consideravelmente a nodulação, além de promover efeito negativo sobre a microbiota do solo. Apesar de todas as três formulações testadas apresentarem algum efeito negativo sobre as características avaliadas da soja, R. Transorb se destaca como a menos indicada para aplicação sobre plantas de soja tolerantes ao glyphosate. Ainda, a formulação Roundup Ready, registrada para a soja transgênica no Brasil, não deve ser aplicada nessa cultura em doses elevadas, visto ser capaz de alterar o teor de alguns nutrientes nas folhas da cultura, entre eles $\mathrm{N}, \mathrm{Ca}, \mathrm{Mg}, \mathrm{Fe}$ e $\mathrm{Cu}$.

\section{AGRADECIMENTOS}

Ao Conselho Nacional de Desenvolvimento Científico e Tecnológico (CNPq), pelo apoio financeiro, e à Coodetec, pelo fornecimento das sementes de soja.

\section{LITERATURA CITADA}

ANDERSON, J. P. E. Soil respiration. In: PAGE, A. L.; MILLER, R. H.; KEENEY, D.R. (Eds.). Methods of Soil Analysis - part 2 - Chemical and microbiological properties. Madison. Agronomy Society of Agronomy, 1982. p. 831871 .

ANDERSON, T. H.; DOMSCH, K. H. Determination of ecophysiological maintenance carbon requirements of soil microorganisms in a dormant state. Biol. Fertil. Soils, v. 1, p. 81-89, 1985 .

DUKE, S. O. et al. Influence of glyphosate on uptake and translocation of calcium ion in soybean seedlings. Weed Res., v. 23, p. 133-139, 1983.

EMPRESA BRASILEIRA DE PESQUISA AGROPECUÁRIA - EMBRAPA. Tecnologia de produção de soja - Paraná - 2002. Londrina: 2002. 195 p. (Embrapa Soja. Sistemas de Produção, 6).

EVENHUIS, B.; WAARD, P. W. F. Principles and practices in plant analysis. In: FAO Soils. Rome: 1980. p. 152-163. (FAO. Bulletin, 38/1).

ISLAM, K. R.; WEIL, R. R. Microwave irradiation of soil for routine measurement of microbial biomass carbon. Biol. Fertil. Soils, v. 27, p. 408-416, 1998.

JAKELAITIS, A. et al. Controle de Digitaria horizontalis pelos herbicidas glyphosate, sulfosate e glyphosate potássico submetidos a diferentes intervalos de chuva após a aplicação. Planta Daninha, v. 19, n. 2, p. 279-285, 2001. 
LI, J. et al. Influence of formulation and glyphosate salt on absorption and translocation in three annual weeds. Weed Sci., v. 53, p. 153-159, 2005.

MALAVOLTA, E.; VITTI, G. C.; OLIVEIRA, S. A. Princípios, métodos e técnicas de avaliação do estado nutricional. In: MALAVOLTA, E.; VITTI, G. C.; OLIVEIRA, S.A. Avaliação do estado nutricional da planta: princípios e aplicações. 2.ed. Piracicaba: POTAFÓS, 1997. p. 115-230.

MARTIN-PREVEL, P.; GAENARD, J.; GAUTIER, P. $L$ 'analyse vegetable dans le controle de l'alimentation des plantes temperées et tropicales. Paris, Techn. Docum., 1984. $802 \mathrm{p}$.

MOLIN, W. T.; HIRASE, K. Comparison of commercial glyphosate formulations for control of prickly sida, purple nutsedge, morningglory and sicklepod. Weed Biol. Manag, v. 4, p. 136-141, 2004.

MOLIN, W. T.; HIRASE, K. Effects of surfactants and simulated rainfall on the efficacy of the Engane formulation of glyphosate in johnsongrass, prickly sida and yellow nutsedge. Weed Biol. Manag., v. 5, p. 123-127, 2005.

PADGETTE, S. R. et al. New weed control opportunities: development of glyphosate-tolerant soybeans. In: DUKE, S. O. (Ed.). Herbicide resistant crops. Boca Raton: CRC, 1995. p.54-80.

PLINE, W.A.; WU, J.; HATZIOS, K. K. Effects of temperature and chemicals additives on the response of transgenic herbicide-soybeans to glufosinate and glyphosate applications. Pest. Biochem. Physiol., v. 65, p. 119-131, 1999.

RAO, I. M.; SHARP, R. E.; BOYER, J. S. Leaf magnesium photosynthetic response to low water potential in sunflower. Plant Physiol., v. 84, p. 1214-1219, 1987.
REDDY, K. N.; ZABLOTOWICZ, R. M. Glyphosateresistant soybean response to various salts of glyphosate and glyphosate accumulation in soybean nodules. Weed Sci., v. 51, p. 496-502, 2003.

RIECHERS, D. E. et al. Surfactant-increased glyphosate uptake into plasma membrane vesicles isolated from common lambsquarters leaves. Plant Physiol., v. 105, p. 1419-1425, 1994.

RODRIGUES, B. N.; ALMEIDA, F. S. Guia de herbicidas. 5.ed. Londrina: Grafmarke, 2005. 591 p.

SANTOS, J. B. et al. Tolerance of Bradyrhizobium strains to glyphosate formulations. Crop Protec., v. 24, p. 543-547, 2005.

SANTOS, J. B. et al. Action of two herbicides on the microbial activity of soil cultivated with common bean (Phaseolus vulgaris) in conventional-till and no-till systems. Weed Res., v. 46, p. 1-6, 2006.

SFREDO, G. J. et al. Soja: nutrição mineral, adubação e calagem. Londrina: EMBRAPA-CNPSO, 1986. 51 p. (EMBRAPA-CNPSO. Documentos, 17).

SHANER, D.; BRIDGES, D. Inhibitors of aromatic amino acid biosyntesis (glyphosate). In: HERBICIDE action course. West Lafayette: Purdue University, 2003. p. 514-529.

SHERRICK, S. L.; HOLT, J. A.; HESS, F. D. Absorption and translocation of MON 0818 adjuvant in field bindweed (Convolvulus arvensis). Weed Sci., v. 34, p. 817-823, 1986.

STOCK, D.; HOLLOWAY, P. J. Possible mechanism for surfactant - induced foliar uptake of agrochemicals. Pestic. Sci., v. 38, p. 165-177, 1993.

VANCE, E. D.; BROOKES, P. C.; JENKINSON, D.S. An extraction method for measuring soil microbial biomass $\mathrm{C}$. Soil Biol. Biochem., v. 19, p. 703-707, 1987. 